\title{
A Case Report on Physiotherapy Rehabilitation of Patient with Lacunar Infarct
}

\author{
Dushyant Bawiskar', Neha Chitale', Waqar Naqvi² and Gaurav Mishra ${ }^{3}$ \\ ${ }^{1}$ Ravi Nair Physiotherapy College, Datta Meghe Institute of Medical Sciences, Wardha, Maharashtra, India. \\ ${ }^{2}$ Professor and Head, Department of Community Physiotherapy, Ravi Nair Physiotherapy College; Research \\ Consultant, Jawaharlal Nehru Medical College, Datta Meghe Institute of Medical Sciences, Wardha, Maharashtra, \\ India. \\ ${ }^{3}$ Department of Radiodiagnosis, Jawaharlal Nehru Medical College, Datta Meghe \\ Institute of Medical Sciences, Sawangi (M), Wardha \\ Corresponding author email: waqar.naqvi@dmimsu.edu.in
}

\section{ABSTRACT}

Stroke is acute episode of a focal neurological deficit lasting more than 24 hours presenting as sudden loss of neurological function caused by an interruption of the blood flow to the brain. Stroke may be ischaemic or haemorrhagic. The haemorrhagic stroke is mostly due to a sudden collapse or secondary trauma of the blood vessels or aneurysm. The stroke of the adult population is a chronic, serious disease. Stroke is the second leading cause of death and adult disability worldwide, and is a global health concern. Infarct of Lacunar in depths of the brain or brain stem are small ischaemic lesions.The lacunar type is a quarter of any ischaemic stroke. A 62 years old female who was a known case of diabetes mellitus, hypertension and some psychiatric illness and was on medication for 2 years, was referred from private hospital with chief complaints of multiple episodes of vomiting and altered sensorium since 2 days. She had weakness in right upper and lower limbs withpain in the right shoulder, aphasia, disturbed balance, coordination and cognition. MRI and CT- SCAN was done. MRI brain revealed Acute lacunar infarcts in bilateral cerebral peduncles, pons, might middle cerebellar peduncle and right cerebellar hemisphere. Also ischemic changes in small vessels were noted. Therapeutic interventions:Medicinal interventions were done primarily followed by physiotherapy interventions which included ice-stroking and functional electrical stimulation to the right side. For left side, active assisted exercises were given. Improvement in muscle tone, strength and balance were reported after successful physical therapy. This case report provides a comprehensive weekly rehabilitation protocol for a patient to gain basic ADLs. Outcome measures were markedly improved with regular exercise and rehab protocol.

KEY WORDS: CVE, QUADRIPLEGIA, LACUNAR INFARCT.

\section{INTRODUCTION}

The acute onset of a focal neurological deficit lasting more than 24 hours is known as a stroke. It is also known

Biosc Biotech Res Comm P-ISSN: 0974-6455 E-ISSN: 2321-4007

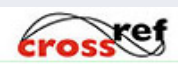

Identifiers and Pagination

Year: 2021 Vol: 14 No (6) Special Issue

Pages: $01-04$

This is an open access article under Creative

Commons License Attribn 4.0 Intl (CC-BY).

DOI: $h t t p: / / d x$.doi.org/10.21786/bbrc/14.6.1

\section{Article Information \\ Received: $29^{\text {th }}$ March 2021}

ccepted after revision: $28^{\text {th }}$ May 2021 as paralysis or apoplexy. It is also known as stroke. The first 24-hour duration after a stroke is an acute stroke. Focal neurological deficit, which persists for less than 24 hours, is not within the scope of this discussion paper, normally known as transient ischaemic attacks (TIA). Stroke is graded as ischaemic $(87 \%)$ or haemorrhagic on the basis of its etiology. Cerebral artery (thrombotic or atherosclerotic (50 percent), embolic (25 percent) and micro artery occlusion (lacunar stroke), ( 25 percent) are the cause of ischaemic strokes. The haemorrhagic stroke is mostly due to a sudden collapse or secondary trauma of the blood vessels or aneurysm (Chugh, 2019). 
The stroke of the adult population is a chronic, serious disease (Fong, 2001). Stroke is the second leading cause of death and adult disability worldwide, and is a global health concern (Bonita, 2004). Infarct of Lacunar in depths of the brain or brain stem are small ischaemic lesions (Caplan and Lacunar, 2014). The lacunar type is a quarter of any ischaemic stroke. Lacunar infarction is the occlusion of a rare thin, perforating artery supplying the subcortia of the brain that causes small infarcts (2-20 $\mathrm{mm}$ of diameter) in deeper cerebral white matter, basal ganglia or pons. While a known stroke form has been in the pipeline for more than 50 years, it remains under consideration whether the cause ischaemic lacunar stroke is different than cortical ischaemic stroke. Lacunar stroke is also not benign, 30\% of patients remain dependent, 5 and scant long term data indicate a second stroke of 5 years for up to $25 \%$. Therefore it may be less than desirable to avoid and treat this particular form of Stroke (Wardlaw, 2005).

Patient Information: A 62 years old female resident of Wardha known case of diabetes mellitus, hypertension and some psychiatric illness and on medication for 2 years, referred from private hospital with chief complaints of multiple episodes of vomiting since 2 days, altered sensorium since 2 days, weakness in right upper and lower limbs since morning. Patient was apparently alright 2 days back when She developed vomiting non projectile, non-bilious, non-blood stained, containing food particles. She also developed altered sensorium in the form of not responding to command. She then developed weakness in right upper and lower limbs. She also had slurring of speech which was associated with difficulty in swallowing.

On examination patient was average, unconscious, disoriented, afebrile, P-62/min., BP142/90 mm Hg, RR16/min, anicteric, JVP not raised, no dehydration, no cervical lymphadenopathy, no cyanosis, no clubbing, pedal oedema. CNS higher function normal, Power cannot be elicited as patient is not responding to commands. Right upper limb-Tone reduced, Power 1/5, reflexes grade 1 , right lower limb. Tone reduced, Power $1 / 5$, Reflexes grade 1 -Right planter extensor, left planter flexor. CVS S1 S2 heard, no murmur-PA soft, non-tender, no organomegaly. RS bilateral equal air entry equal, no crackles. On Investigation she had hypernatremia. Her hemogram Normocytic normochromic RBCs. WBCs show neutrophil leucocytosis. Platelets adequate, bilirubin normal, creatinine and liver enzymes were normal. Her Ct head is suggestive as: - Chronic lacunar infarct in left lentiform nucleus and splenium of corpus callosum. Age related brain parenchymal changes. Her MRI brain which suggested Acute lacunar infarcts in bilateral cerebral peduncles, pons, might middle cerebellar peduncle and right cerebellar hemisphere.

Chronic lacunar infarcts in bilateral putamen and left corona radiata. Small vessel ischemic changes (Fazekas grade IJ lesions). Patient managed with antiplatelets, statins, antihypertensive, oral hypoglycaemic agents and other supportive treatment, Physiotherapy given. The nature, course and prognosis of the patient's condition are explained to the relatives Feeding through Ryles tube and physiotherapy were taught to the relative -Patient discharged with vital stable and advised to follow up in OPD after 1 month.

Clinical Finding: After admission to physiotherapy OPD (Day 1), a comprehensive examination was conducted. Original Initial. The mental status assessment could not be carried out because of communication problems for the patient. It's in a trouble because there was difficulty in. In receptive language awareness and disability. Sensory testing was also limited and secondary to inability to communicate. Motor evaluation: Complete spasticity, joint play and compliance with soft tissue have been evaluated. The scale of engine assessment was used for engine feature assessment. Spasticity evaluation: Spasticity was measured by using the Modified Ashworth scale. Spasticity was grade 1 for shoulder pads, elbow pads, wrist flexors and hip flexors, and grades 1+ for knee and ankle flexors. Plantar flexors Spasticity was 0 for the extending category of muscles. Reflex: Each reflex was natural, i.e. 2+. Gait: The patient could not rise and go.

Diagnostic Methods For the diagnosis of problems associated with patient's day to day life including the activities of daily living and motor function, Motor Assessment Scale was used. Basic mobility activities were assessed using STREAM, FIM, which further helped to design the rehabilitation program and achieve the functional goals. Motor assessment scale - On Motor assessment scale patient total score was 8 on 1st day. FIM - On FIM scale patient total score was 18 on 1st day. STREAM - on this scale patient scored minimal points on 1st day. Diagnostic challenges Patient's lack of education of the condition and ignorance led to altered prognosis. Patient's denial for the check-up and tests led to stroke. Major challenge was communication with the patient, which was due to comprehension problem. Later, the patient started responding through gestures. Shoulder subluxation was also a challenge as it delayed the recovery.

\section{Management}

Phase 1(Acute Phase)

Phase 1 focuses on Patient education and prevention of secondary complication.

Patient and relatives were explained about the condition and the importance of physiotherapy in rehabilitation. Two hourly positioning was given patient was log rolled on both the sides and in supine position in order to prevent pressure sores. Positioning was done to avoid contractures. Passive ankle toe movement was given in order to maintain distal circulation and DVT pump was advised. Segmental breathing exercises were given for lower lobe and middle lobe in order to maintain the perfusion and ventilation ratio. Passive movements were given to maintain the range of motion of all the joints. 
Phase 2:-

Phase 2 was focused on advancement in phase one and on managing the spasticity, improving the motor learning.

Early mobilisation was given and elongation of spastic muscle with sustained stretch through positioning was done. Prolong pressure on spastic muscle was also given in order to induce inhibitory effect and slow rocking movements were given to increase inhibitory effect through adding influence over vestibular stimulation. Proprioceptive neuromuscular facilitation was given for upper trunk to maintain reduced muscle tone. Motor learning was promoted using strategy development, feedback and practice. First the patient was assisted in learning the task. Then the task was demonstrated and then the patient was asked to practice for the same.

Phase 3:-

Phase 3 was focused on advancement in phase 2 and on improving postural control and functional mobility.

Functional training activities like Rolling, Sitting, and Bridging were taught. Upper limb PNF patterns were used to enhance the movement. Training emphasised on proper symmetrical posture with proper spine and pelvic alignment. Sitting was progressed from static to dynamic to dynamic by giving reach outs. Bridging was given in order to relief pressure over the buttocks area and to initiate bed mobility.

Phase 4:-

Phase 4 focused on balance and gait training

Symmetric weight bearing was promoted. Patient was taught to maintain the Centre of mass within the base of support. Destabilizing functional activities were given in order increase the difficulty level destabilizing exercises included sit-to- stand, flooring to standing. Dual task training was given in order to improve balance.

Locomotive training was given by explaining the Patient normal kinematics and phase relationship in gait cycle, assistance was given by the therapist. For Gait training patient was encouraged to take equal steps and maintain the natural rhythm of walking and speed. Walking in natural environment was promoted. Ankle foot orthosis was given for stabilisation. Practice was given for functional or task specific skills like backward walking, forward walking and side walking. Follow up and outcomes Patient was assessed on day 1, at 4th week, 8th week and 12th week of intervention. Spasticity Spasticity changes were grade 1 on day 1 , grade $1+C t$ 2 in the 4 th week, 1 in 8 th week and 0 in 12 th week. Reflex- Reflexes were $2+$ on day $1,3+$ in 4 th week, 2+ \& $3+$ (TA \& Knee jerk) in 8th week and 2+ in 12th week. Motor Assessment Scale- Scores were 8, 14, 20 and 27 in respective weeks. FIM score- Scores were 18, 31, 41 and 47 in respective weeks.

\section{DISCUSSION}

The patient had cognitive difficulties, i.e. comprehension because of a sudden coronary episode leading to multiple communication problems. The patient cannot understand what he says and he often has trouble understanding and following the directions. The patient can only sound like 0000 and pinch in pain. Speaking to the family about this issue, they told the family that they could only use a method of removal and read his hand movements to understand what he meant.

In this case patients attended physiotherapy, complaining of being unable to perform tasks on the right side of the body including the right lower and upper limbs, trouble with ADLs, incapacity to speak, failure to stand and move because of fatigue after a stroke. Multiple treatment methods such as icing (cryotherapy) approaches by spastic muscles have been planned that have shown to decrease spastic muscle resistance clinically to quick deepening and decreased or inhibit the Clonus( Monaghan et al., 2017).

Electrical muscle stimulation was used to reinforce or activate the weak muscle and re-learn the motor after destroying the central nervous system. Traditional therapy requires workouts, resisted exercises and stretching. exercises It plays as a key recovery factor, preventing muscle atrophy. The extension is incorporated to maintain or improve the joint mobility by affecting the expansion of the soft tissue of the joints( $\mathrm{Wu}$ et al., 2019). A number of related studies were reviewed (Bawiskar et al., 2019; Khanna et al., 2019; Gugulothu et al., 2019; Chiwhane et al., 2018; Khatib et al., 2017). Many studies from Global Burden of Disease study reflect the evidences of similar problems(Murray et al., 2020; Vos et al., 2020; Wang et al., 2020; Lozano et al., 2020). Studies by Khairkar et al.(2012) , Chaturvedi et al.(2020) , Mishra et al. (2020) , Pratapa et al. (2020) , Zade et. al. (2020) were reported on strokes in different contexts.

\section{CONCLUSION}

This article is not much frequently publishing model regards "Chronic lacunar infarcts in bilateral putamen and left corona radiata.”. This case report demonstrated that the patient achieved maximum goal. This case report provided a comprehensive weekly rehabilitation protocol for a patient to gain basic ADLs. Outcome measures were markedly improved with regular exercise and rehab protocol.

\section{REFERENCES}

Bawiskar, N., Kothari, N. , Kumar, S., Acharya, S., Chaudhari, S.S. 2019. Clinico-Radiological Association of Serum Calcium, Ionic Calcium and Albumin Corrected Serum Calcium in Acute Ischaemic Stroke.. International Journal of Pharmaceutical Research, 11(3),pp.144548.

Bonita, R., Mendis, S., Truelsen, T., Bogousslavsky, J., Toole, J. and Yatsu, F., 2004. The global stroke initiative. The Lancet Neurology, 3(7), pp.391-393.

Caplan, L.R., 2015. Lacunar infarction and small vessel 
disease: pathology and pathophysiology. Journal of stroke, 17(1), p.2.

Chaturvedi, S., Garikapati, A., Ansari, I., Bagga, C. and Kumar, S., 2020. Trastuzumab induced cardiomyopathy with cerebellar stroke: Double Trouble. Medical Science, 24(104), pp.2424-2427.

Chiwhane, A., Pradeep. 2018. Study of Rhythm Disturbances in Acute Myocardial Infarction.Journal of Association of Physicians of India, 66, pp. 54-58.

Chugh, C. 2019. Acute Ischemic Stroke: Management Approach. Indian J Crit Care Med. 2,S140-6.

Fong, K.N., Chan, C.C. and Au, D.K., 2001. Relationship of motor and cognitive abilities to functional performance in stroke rehabilitation. Brain Injury, 15(5), pp.443453.

Gugulothu, S.S., Choudhary, R.S., Telrandhe, S. 2019. CMOS Amplifiers Design for Neural Recording System." Journal of Advanced Research in Dynamical and Control Systems, 11(8), pp. 3071-76.

Khairkar, P. and Diwan, S., 2012. Late-onset obsessivecompulsive disorder with comorbid narcolepsy after perfect blend of thalamo-striatal stroke and poststreptococcal infection. The Journal of neuropsychiatry and clinical neurosciences, 24(4), pp.E29-E31.

Khanna, S., Inamdar, A., Kumar, S. and Basat, A.V., Study of Serum Uric Acid Levels in Acute Stroke. International Journal of Pharmaceutical Research, 11, pp.2041-44.

Khatib, M.N., Kirubakaran, R., Gaidhane, S., Shankar, A.H. and Syed, Z.Q., 2017. Yoga for improving functional capacity, quality of life and cardiovascular outcomes in people with heart failure. The Cochrane Database of Systematic Reviews, 2017(7).

Latchoumi, T.P., Ezhilarasi, T.P. and Balamurugan, K., 2019. Bio-inspired weighed quantum particle swarm optimization and smooth support vector machine ensembles for identification of abnormalities in medical data. SN Applied Sciences, 1(10), pp.1-10.

Lozano, R., Fullman, N., Mumford, J.E., Knight, M., Barthelemy, C.M., Abbafati, C., Abbastabar, H., AbdAllah, F., Abdollahi, M., Abedi, A. and Abolhassani, H., 2020. Measuring universal health coverage based on an index of effective coverage of health services in 204 countries and territories, 1990-2019: a systematic analysis for the Global Burden of Disease Study 2019. The Lancet, 396(10258), pp.1250-1284.

Mishra, S., Palak, D., Waqar, M., Naqvi, Arti Sahu. 2020. Regaining Activities of Daily Living in Patient with
Middle Cerebral Artery Stroke- A Case Report. MEDICAL SCIENCE , 24(103), pp.1731-37.

Monaghan, K., Horgan, F., Blake, C., Cornall, C., Hickey, P.P., Lyons, B.E. et al. 2017. Physical treatment interventions for managing spasticity after stroke. Cochrane Database Syst Rev, (2).

Murray, C.J., Abbafati, C., Abbas, K.M., Abbasi, M., Abbasi-Kangevari, M., Abd-Allah, F., Abdollahi, M., Abedi, P., Abedi, A., Abolhassani, H. and Aboyans, V., 2020. Five insights from the global burden of disease study 2019. The Lancet, 396(10258), pp.1135-1159.

Murray, C.J., Aravkin, A.Y., Zheng, P., Abbafati, C., Abbas, K.M., Abbasi-Kangevari, M., Abd-Allah, F., Abdelalim, A., Abdollahi, M., Abdollahpour, I. and Abegaz, K.H., 2020. Global burden of 87 risk factors in 204 countries and territories, 1990-2019: a systematic analysis for the Global Burden of Disease Study 2019. The Lancet, 396(10258), pp.1223-1249.

Pratapa, S., Sourya A., Swapnil L., Amol A.. 2020. Locked-in State with Ondine's Curse in a Case of Vertibro Basilar Territay Stroke. JOURNAL OF CLINICAL AND DIAGNOSTIC RESEARCH, 14(3) https://doi. org/10.7860/JCDR/2020/43776.13592.

Vos, T., Lim, S.S., Abbafati, C., Abbas, K.M., Abbasi, M., Abbasifard, M., Abbasi-Kangevari, M., Abbastabar, H., Abd-Allah, F., Abdelalim, A. and Abdollahi, M., 2020. Global burden of 369 diseases and injuries in 204 countries and territories, 1990-2019: a systematic analysis for the Global Burden of Disease Study 2019. The Lancet, 396(10258), pp.1204-1222.

Wang, H., Abbas, K.M., Abbasifard, M., AbbasiKangevari, M., Abbastabar, H., Abd-Allah, F., Abdelalim, A., Abolhassani, H., Abreu, L.G., Abrigo, M.R. and Abushouk, A.I., 2020. Global age-sex-specific fertility, mortality, healthy life expectancy (HALE), and population estimates in 204 countries and territories, 1950-2019: a comprehensive demographic analysis for the Global Burden of Disease Study 2019. The Lancet, 396(10258), pp.1160-1203.

Wardlaw, J.M., 2005. What causes lacunar stroke?.

Wu, C., Liu, Y. and Ji, X., 2019. Letter by Wu et al Regarding Article,"Risk Factors for Acute Ischemic Stroke Caused by Anterior Large Vessel Occlusion". Stroke, 50(8), pp.e237-e237.

Zade, R., Priyanka S., Gunjan S., Pratik P., Ragini Dadgal. 2020. Comprehensive Physical Therapy Improves Functional Recovery in a Rare Case of Stroke Associated with Asthma: A Case Report. MEDICAL SCIENCE, 24(105), pp. 2893-99. 